\title{
Voltage-controlled phase matching in quadrupole second-harmonic generation
}

\author{
S. V. Lazarenko,* A. Kirilyuk, and Th. Rasing \\ Institute for Molecules and Materials, Radboud University Nijmegen, Toernooiveld 1, 6525 ED Nijmegen, The Netherlands
}

(Received 11 July 2006; published 16 October 2006)

\begin{abstract}
We have observed strong phase-matched (PM) quadrupole optical second-harmonic generation (SHG) from nematic liquid crystals that possess inversion symmetry. PM conditions were achieved for two configurations by voltage control of the molecular orientation and thus of the refractive indices. Quadrupole SHG was separated from electric-field-induced dipole-type SHG with the help of time-gated measurements in a pulsed electric field.
\end{abstract}

DOI: 10.1103/PhysRevE.74.045601

PACS number(s): 42.70.Mp, 42.70.Df, 42.65.-k, 78.20.-e

For efficient optical second-harmonic generation (SHG) in a material, a polar ordering is needed [1]. In such a case the material structure is noncentrosymmetric and the SHG signal is allowed in the electric-dipole approximation. To achieve a high efficiency, it is important that the incident fundamental and the generated second-harmonic waves propagate through the medium in phase. Because of the usual dispersion of the refractive index, phase matching (PM) can normally only be obtained in certain birefringent materials $[1,2]$. Thus, although PM is widely used for studying materials with broken symmetry $[3,4]$, little attention has been paid to the class of materials where the SHG signal has a quadrupole origin.

Because nematic liquid crystal (NLC) molecules are oriented antiparallel to each other in the bulk $[5,6]$, nematics have inversion symmetry and are generally not expected to show strong nonlinear optical properties. However, the large molecular nonlinear optical susceptibility of individual molecules [7] made the SHG technique a very useful tool for the study of the surface alignment of NLC molecules $[6,8]$. The relatively small but nonzero bulk contribution originates mainly from the electric-quadrupole part in the induced nonlinear polarizability [9], but in some cases could still be significant in comparison with the electric-dipole contribution from the surface [10]. In addition, an applied electric field can induce dipolar ordering and thus also bulk electric-dipole SHG, which has been used to study NLC cells under applied voltage [11-13].

This Rapid Communication describes the observation of voltage-controlled PM SHG of quadrupole origin from nematic LC cells. The effect of the applied voltage is both a matching of the refractive indices via molecular reorientation and inducing an extra contribution to the SHG. Therefore, to separate the real quadrupole SHG contribution from this extra electric field-induced SHG (EFISHG) signal, timeresolved measurements were used. Depending on the polarization combination, both type-I and -II PM [1,2] were observed. The possibility to use this observation for studying the molecular reorientation in NLC cells is also discussed.

The samples used for the experiments were parallelaligned E63 NLC cells with a thickness of 20 and $50 \mu \mathrm{m}$. The LC mixture was held between two glass substrates cov-

\footnotetext{
*Electronic address: S.Lazarenko@ science.ru.nl
}

ered with $20 \mathrm{~nm}$ conductive indium tin oxide (ITO). On top of ITO, a rubbed polymer material PI2555 with a low pretilt angle was used to impose a high anchoring potential on the NLC molecules at the cell surfaces parallel to the $x$ axis [see Fig. 1(a)].

For the SHG measurements, a pulsed-laser beam from a Ti-sapphire laser (76-MHz 100-fs pulses) with a wavelength of $800 \mathrm{~nm}$ was focused onto the sample in a $200-\mu \mathrm{m}$ spot using a lens with a focal distance of $200 \mathrm{~mm}$. The average laser intensity was $20 \mathrm{~mW}$. The polarization of the incoming fundamental laser beam could be varied using a Babinet Soleil compensator in combination with a Glan-Taylor polarizer to ensure a high degree of light polarization. An analyzer was set to choose the polarization of the outgoing SHG, which was detected after proper filtering with a photomultiplier. It was verified by spectral and intensity measurements that only second-harmonic light was generated. The linear optical measurements were performed with the same laser, two crossed polarizers, a rotational stage with the sample

a)
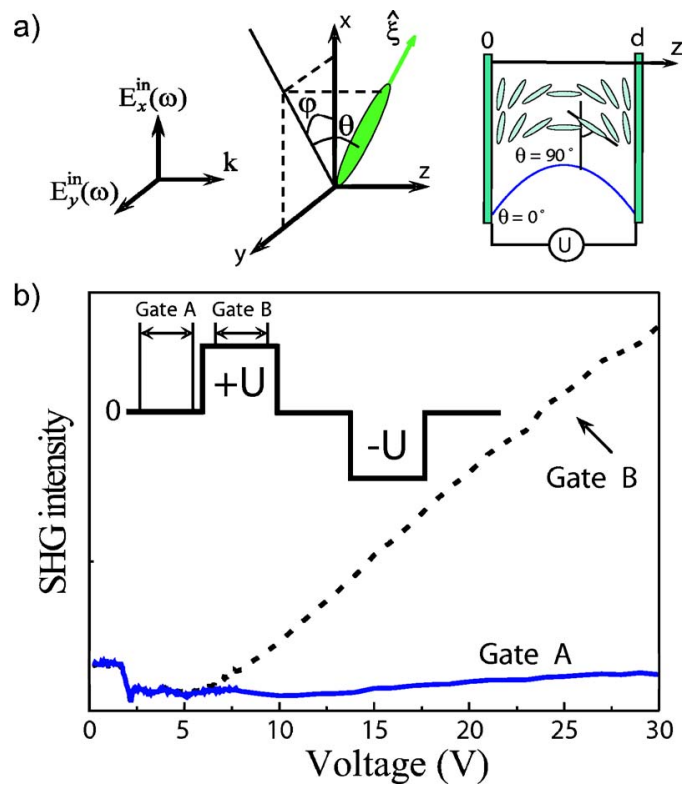

FIG. 1. (Color online) Experimental configuration: (a) sample geometry, $\varphi$ is the azimuthal angle and $\theta$ is the angle between substrate and orientation of the director; (b) the form of rectangular balanced voltage and experimental data for a 50- $\mu$ m-thick sample, $E_{x}^{\text {in }} E_{x}^{\text {out }}$ polarization combination. Gate $A$ field ofF, gate $B$ field oN. 
holder, and a photodiode. The coordinate frame was chosen so that the top glass-NLC interface is in the $x y$ plane while the director of the NLC in this plane is pointed along the $x$ axis. The electromagnetic field is therefore propagating along the positive $z$ axis; see Fig. 1(a).

$E_{x}$ indicates the polarization parallel to the $x$ axis, $E_{y}$ the polarization parallel to the $y$ axis, and $E_{45}$ the polarization at $\pm 45^{\circ}$ from the $x$ axis, in the $x y$ plane.

The nonlinear optical response of a medium is described by the polarization $\mathbf{P}$ induced by a strong electromagnetic field. The induced polarization at the double frequency can be written as $[8,15]$

$$
\begin{aligned}
\mathbf{P}(2 \omega)= & \boldsymbol{\chi}^{(d)} \cdot \mathbf{E}(\omega) \mathbf{E}(\omega)+\boldsymbol{\chi}_{e}^{(d)} \cdot \mathbf{E}(\omega) \mathbf{E}(\omega) \mathbf{E}(0) \\
& +\boldsymbol{\chi}_{1}^{(q)} \cdot \mathbf{E}(\omega) \boldsymbol{\nabla} \cdot \mathbf{E}(\omega)+\left(\boldsymbol{\nabla} \cdot \boldsymbol{\chi}_{2}^{(q)}\right) \mathbf{E}(\omega) \mathbf{E}(\omega) .
\end{aligned}
$$

In this equation $\mathbf{E}(\omega)$ is the fundamental field inside the medium, $\boldsymbol{\chi}^{(d)}$ is the electric-dipole-type second-order susceptibility tensor of rank 3 , and $\boldsymbol{\chi}_{1}^{(q)}$ and $\boldsymbol{\chi}_{2}^{(q)}$ are the electricquadrupole susceptibilities, both of rank 4. $\boldsymbol{\chi}_{e}^{(d)}$ describes EFISHG. For materials with inversion symmetry $\boldsymbol{\chi}^{(d)} \equiv 0$.

A NLC is composed of rodlike molecules with the long axis of the molecules pointing along a common direction, the director, while they do not posses positional order [14]. NLC's are therefore centrosymmetric and electric-dipole SHG vanishes.

In the bulk of a homogeneous material $\boldsymbol{\nabla} \cdot \boldsymbol{\chi}_{2}^{(q)}$ is obviously zero and can be neglected. However, near surfaces and interfaces this term can be significant. In addition, a considerable contribution from this term can be expected during the process of molecular reorientation due to strong inhomogeneities of the director across the LC cell.

When an external voltage is applied to an NLC cell, the electric-dipole EFISHG term $\left(\boldsymbol{\chi}_{e}^{(d)}\right)$ may dominate the weaker quadrupole terms. To avoid this, the voltage was applied in a rectangular balanced form [see the inset in Fig. 1(b)], with a frequency of $5 \mathrm{kHz}$. Such a temporal shape of the voltage does not affect the reorientation of the molecules, because the latter is even with respect to the electric field. However, it allows us to distinguish between the state with electric field ON [inset in Fig. 1(b), gate $B$ ] and field OFF (gate $A$ ), for the same orientation of the molecules. This is possible because the molecular reorientation response is much slower than the rise and decay of the electric-fieldinduced SHG signal [15]. Therefore, in the OFF state, a purely quadrupole contribution is detected.

For these measurements we used two photoncounters SR400 in gated mode using different delay times and a gate width of $40 \mu \mathrm{s}$. If $\mathbf{E}=\mathbf{0}$, gate $A$ in Fig. 1(b), the SHG signal has a quadrupole origin (no EFISHG contribution) and a change of the intensity is purely due to the molecular reorientation. For a delay time of $\tau=55 \mu \mathrm{s}$, gate $B$, an additional contribution induced by the positive voltage was present and for $\tau=155 \mu$ s a contribution induced by the negative voltage. From these experimental data we can conclude that the EFISHG signal gives a significant contribution at voltages higher than $5 \mathrm{~V}$, but only for the $E_{x}^{\text {in }} E_{x}^{\text {out }}$ experimental
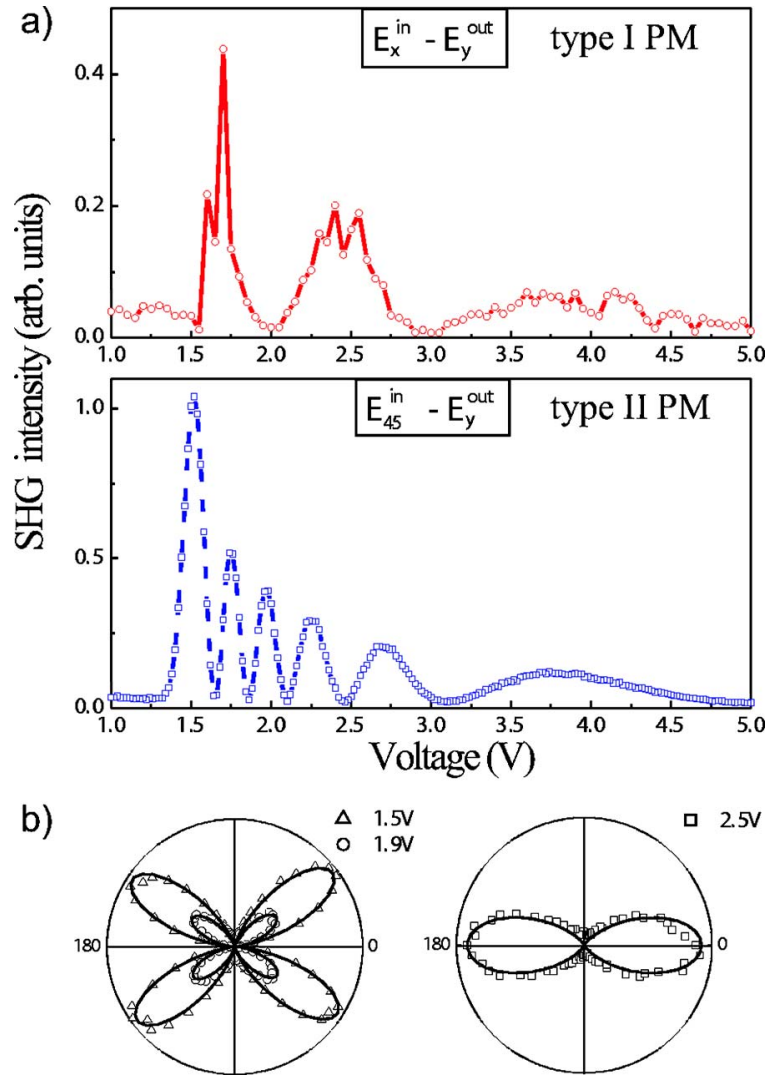

FIG. 2. (Color online) (a) SHG intensity as a function of the applied voltage amplitude for $E_{x}^{\text {in }} E_{y}^{\text {out }}$ and $E_{45}^{\text {in }} E_{y}^{\text {out }}$ polarization combinations; (b) the SHG rotational anisotropy patterns at different voltages for crossed polarization combinations. At $0^{\circ}$ the polarizer is pointing along the director and the analyzer perpendicular to it. Solid curves are fits by Eqs. (5) and (6).

configuration-i.e., when both fundamental and SHG polarizations are parallel to the NLC director. There was no EFISHG signal detected with $E_{y}^{\text {out }}$ polarization-i.e., parallel to the short optical axis of the NLC molecules.

The changes in the $E_{y}^{\text {out }}$ polarization (quadrupole SHG) induced by the molecular reorientation are drastically different. In contrast to the steplike decrease of the SHG intensity shown in Fig. 1(b), oscillations of very large amplitude are observed [see Fig. 2(a)] for the outgoing SHG polarized perpendicular to the molecular axis. A similar and even stronger effect is observed if, in addition, the incoming fundamental polarization is set to $E_{45}^{i n}$. The first peak in the curve for the $E_{45}^{i n} E_{y}^{\text {out }}$ polarization combination is particularly pronounced, demonstrating an increase of the SHG intensity by almost two orders of magnitude.

Such a strong dependence of the SHG intensity for crossed-polarization configurations, together with the absence of such oscillations when the polarizations are set to parallel, gives a strong indication for phase matching in these birefringent samples.

For efficient frequency conversion one must ensure that the phase velocities of the interacting fundamental and second-harmonic waves are equal so that the SHG signal can build up in intensity at the expense of the pump radiation throughout the bulk of the medium $[1,2]$. In a normal disper- 
sive medium the index of refraction at the double frequency is higher than at the fundamental, $n(2 \omega)>n(\omega)$, so the fundamental and $\mathrm{SH}$ waves always travel at different velocities. The continuous phase slip between these waves leads to an oscillatory change of the intensity of the SHG signal, described by

$$
I^{S H G} \propto \chi^{2} I_{0}^{2} d^{2} \frac{\sin ^{2} u}{u^{2}}, \quad u=\Delta n \frac{\omega d}{c},
$$

where $d$ is the thickness of the sample, $\Delta n=n(2 \omega)-n(\omega)$. Phase matching occurs when $\Delta n=0$. In birefringent crystals in principle two types of PM are possible [2]. E63 and the majority of NLC's are positive uniaxial materials with $n_{e}(\omega)>n_{o}(\omega)$, where $e$ and $o$ indices denote extraordinary and ordinary waves, respectively. For such media we can write

$$
\begin{gathered}
\text { type-I PM:ee } \rightarrow o, \quad n_{o}(2 \omega)=n_{e}(\omega), \\
\text { type-II PM:eo } \rightarrow o, \quad n_{o}(2 \omega)=\frac{1}{2}\left[n_{o}(\omega)+n_{e}(\omega)\right] .
\end{gathered}
$$

For type-I PM (two $e$ fundamental waves produce an $o$ wave at double frequency), the effective susceptibility $\chi_{e f f}^{e e \rightarrow o} \propto \chi_{y x x z}^{Q}$, and for the type-II PM, $\chi_{e f f}^{e o \rightarrow o} \propto \chi_{y y x z}^{Q}$. The maximum of the SHG intensity in the case of type-I PM should thus be achieved for the experimental configuration with the incoming beam polarized parallel to the long optical axis $\left(E_{x}^{i n}\right)$ and the analyzer placed perpendicular $\left(E_{y}^{o u t}\right)$ to it. In the case of type-II PM, the incoming polarization should be at $45^{\circ}$ to the director orientation and the analyzer along the short axis. These are exactly the two configurations where the oscillatory enhancement of the SHG intensity is experimentally detected, allowing us to tentatively identify these two types of PM.

An alternative demonstration of the polarizational dependence of SHG can be seen in the SHG rotational anisotropy as a function of voltage-induced molecular reorientation [see Fig. 2(b)]. For the crossed polarization combination, two significantly different patterns are observed for voltages of $1.5 \mathrm{~V}$ and $2.5 \mathrm{~V}$, which correspond to type-II and type-I PM, respectively. At $0^{\circ}$ the polarizer is pointing along the director and the analyzer perpendicular to it. The solid curves in these plots represent theoretical fits by the following formulas:

$$
\begin{gathered}
I_{I}^{S H G} \propto \chi_{y x x z}^{Q} E^{2} \cos ^{6} \varphi, \\
I_{I I}^{S H G} \propto \chi_{y y x z}^{Q} E^{2} \cos ^{4} \varphi \sin ^{2} \varphi,
\end{gathered}
$$

where $\varphi$ is the azimuthal angle [see Fig. 1(a)]. The perfect correspondence of the experimental data to these formulas once again confirms the PM origin of the observed signals.

In a fully aligned E63 mixture the refractive indices at the fundamental $(800 \mathrm{~nm})$ and second-harmonic $(400 \mathrm{~nm})$ wavelengths are $n_{e}(\omega)=1.715, n_{o}(\omega)=1.507, n_{e}(2 \omega)=1.85$, and $n_{o}(2 \omega)=1.55$. This yields $\Delta n=n_{e}(\omega)-n_{o}(2 \omega)=0.208$ which is far away from PM. The birefringence is easily adjusted, however, by reorienting the NLC molecules with an applied voltage.

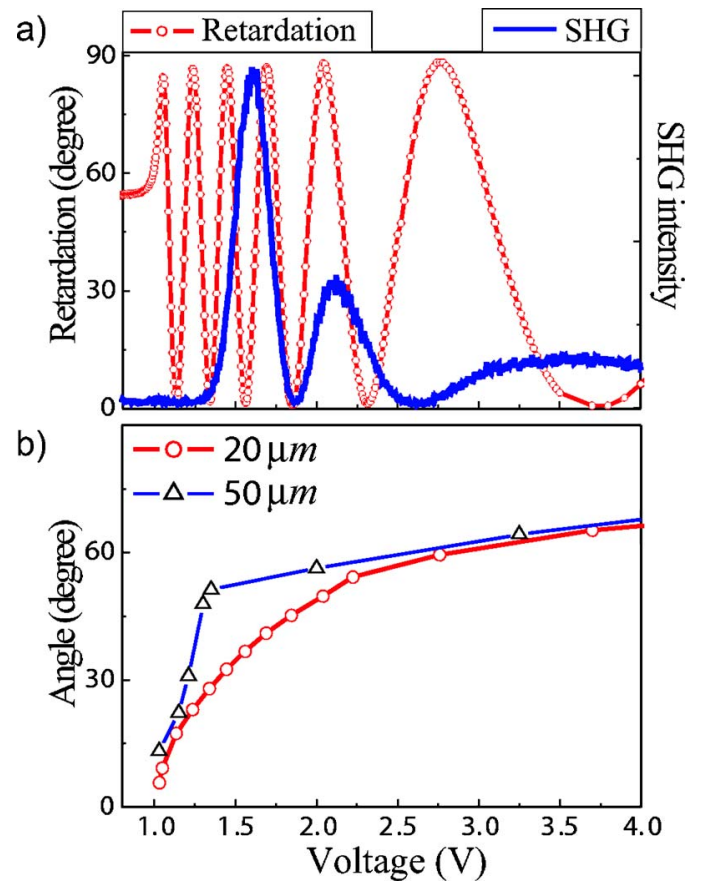

FIG. 3. (Color online) (a) Experimental linear and nonlinear data for $20-\mu \mathrm{m}$-thick sample; (b) calculated angle dependence.

The corresponding change of refractive indices can be written as

$$
n_{o}=n_{\perp}, \quad n_{e}(z)=\frac{n_{\perp} n_{\|}}{\sqrt{n_{\|}^{2} \sin ^{2} \theta(z)+n_{\perp}^{2} \cos ^{2} \theta(z)}},
$$

where $\theta$ is the angle between the substrate and orientation of the director and $n_{e}(z)$ and $\theta(z)$ indicate the $z$ dependence of the $n_{e}$ and $\theta$, respectively. During molecular reorientation the value of $n_{e}$ changes from the initial $n_{e}(\omega)=1.715$ at $0 \mathrm{~V}$ to $n_{e}(\omega)=n_{o}(\omega)=1.507$ at sufficiently high voltage, when all molecules are reoriented. From Eq. (7) we can estimate the value of $\theta$ at which PM will be achieved. Thus, type-I PM should occur at $\theta \approx 60^{\circ}$ and type-II PM at $\theta \approx 45^{\circ}$. By reorientation of the LC molecules in an external electric field, one should first encounter type-II and at higher voltage type-I PM. Figure 2 confirms this prediction: the first peak (at $1.5 \mathrm{~V}$ ) is observed for $E_{45}^{\text {in }} E_{y}^{\text {out }}$, whereas the second one (at $1.7 \mathrm{~V}$ ) is observed for $E_{x}^{\text {in }} E_{y}^{\text {out }}$.

Following the same arguments, all enhanced outgoing SHG signals should be polarized along the short axis $E_{y}^{\text {out }}$. In order to check this we performed the same measurements but with the analyzer along the long axis $E_{x}^{\text {out }}$. No enhancement was observed for this situation.

The phase matching in the NLC cell will also be influenced by the inhomogeneous director profile across the cell thickness. Due to the anchoring energy, the voltage-induced reorientation of the molecules near the surface will be less than in the bulk $[16,17]$. The corresponding $U$-like director profile will lead to a change of the speed of the $e$ wave through the sample while the $o$ wave will not be affected. As a result $\Delta n$ will vary depending on the distance from the surface and consequently it will not be possible to achieve 
exact PM conditions throughout the whole thickness of the sample. This inhomogeneity is a relatively weak effect in thicker samples, which may also explain the fact that in the thinner sample of $20 \mu \mathrm{m}$, only type-II PM has been observed. Numerical simulations showed that even with such a $U$-like profile a high enhancement of the SHG signal should still be observable, as is indeed the case.

The average angle of the molecular reorientation was obtained with the help of simple birefringence measurements. In these linear transmission experiments the NLC cell was placed at normal incidence between crossed polarizers and the transmission of the laser beam $(\lambda=800 \mathrm{~nm})$ was monitored as a function of the applied cell voltage [see Fig. 3(a)]. The directions of the polarizers were at an angle of $\pm 45^{\circ}$ relative to the $x$ axis of the cell. Based on a simple model [16], the averaged director angle at the first enhancement peak could be calculated from the transmission data, yielding $\theta \approx 52^{\circ}$ (see Fig. 3), while for the E63 molecules, type-II PM should ideally be achieved at $\theta \approx 45^{\circ}$. In the case of type-I $\mathrm{PM}$, the expected value is $\theta \approx 60^{\circ}$, but from the linear data we found $\theta \approx 55^{\circ}$. This difference and asymmetric behavior of the SHG intensity, the absence of smaller oscillations before reaching PM conditions, can be explained by the inhomogeneous molecular orientation through the sample.
Although this strong inhomogeneity of molecular reorientation increases the gradient term in the SHG signal, it disturbs the PM conditions. Experimentally measured dependences of the average angle of the molecular reorientation versus applied voltage are rather different for the samples with different thicknesses [see Fig. 3(b)].

In conclusion, a simple and easily controllable way to obtain phase-matched quadrupole SHG in NLC cells was demonstrated. While a NLC cell is one of the most widely used types of liquid crystals, PM is normally observed only in media with broken inversion symmetry such as ferroelectric LC's. The method is based on the electric field control of the molecular orientation and thus the birefringence. Timeresolved measurements with rectangular balanced voltage pulses were used to separate the quadrupole SHG signal from the electric-dipole EFISHG contribution, proving the bulk quadrupolar origin of the phase matched SHG signals.

The authors like to thank Jun $\mathrm{Li}$ and Shin-Tson $\mathrm{Wu}$ from the University of Central Florida for the information about the refractive indices of LC-E63. These studies were supported by the Dutch Technology Foundation (STW), Project No. NNS4857.
[1] Y. R. Shen, The Principles of Nonlinear Optics (Wiley, New York, 1984).

[2] J. E. Midwinter and J. Warner, Br. J. Appl. Phys. 16, 1135 (1965).

[3] I. Drevensek Olenik, R. Torre, and M. Copic, Phys. Rev. E 50, 3766 (1994).

[4] V. S. U. Fazio, S. T. Lagerwall, P. Busson, A. Hult, and H. Motschmann, Appl. Phys. Lett. 77, 319 (2000).

[5] A. J. Leadbetter and A. J. Mehta, Mol. Cryst. Liq. Cryst. 72, 51 (1981).

[6] M. B. Feller, W. Chen, and Y. R. Shen, Phys. Rev. A 43, 6778 (1991).

[7] G. Berkovic, Th. Rasing, and Y. R. Shen, J. Opt. Soc. Am. B 4, 945 (1987).

[8] P. Guyot-Sionnest and Y. R. Shen, Phys. Rev. B 38, 7985 (1988).
[9] R. W. Munn, Mol. Phys. 89, 555 (1996).

[10] P. Guyot-Sionnest, H. Hsiung, and Y. R. Shen, Phys. Rev. Lett. 57, 2963 (1986).

[11] S. K. Saha and G. K. Wong, Opt. Commun. 30, 119 (1979).

[12] S. K. Saha and G. K. Wong, Appl. Phys. Lett. 34, 423 (1979).

[13] M. I. Barnik, L. M. Blinov, A. M. Dorozhkin, and N. M. Shtykov, Zh. Eksp. Teor. Fiz. 81, 1763 (1981) [Sov. Phys. JETP 54, 935 (1981)].

[14] P. G. de Gennes, The Physics of Liquid Crystals (Clarendon, Oxford, 1975).

[15] R. Stolle, T. Durr, and G. Marowsky, J. Opt. Soc. Am. B 14, 1583 (1997).

[16] L. M. Blinov and V. G. Chigrinov., Electro-Optic Effects in Liquid Crystal Materials (Springer, New York, 1994).

[17] I. I. Smalyukh, S. V. Shiyanovskii, and O. D. Lavrentovich, Chem. Phys. Lett. 336, 88 (2001). 